\title{
Attitudes of Nonpalliative Care Nurses towards Palliative Care
}

\author{
Victoria Tait, ${ }^{1}$ Megan Higgs, ${ }^{2}$ Linda Magann, ${ }^{1}$ Joanne Dixon, \\ Jan Maree Davis, ${ }^{1}$ and Ritin Fernandez ${ }^{2,3}$ \\ ${ }^{1}$ St George Hospital, Kogarah, NSW 2217, Australia \\ ${ }^{2}$ Centre for Research in Nursing and Health, St George Hospital, Level 1 James Laws House, Kogarah, NSW 2217, Australia \\ ${ }^{3}$ School of Nursing, University of Wollongong, Northfields Avenue, Wollongong, NSW 2522, Australia
}

Correspondence should be addressed to Megan Higgs; megan.higgs@sesiahs.health.nsw.gov.au

Received 26 September 2014; Revised 1 December 2014; Accepted 11 December 2014

Academic Editor: Luc Deliens

Copyright (C) 2015 Victoria Tait et al. This is an open access article distributed under the Creative Commons Attribution License, which permits unrestricted use, distribution, and reproduction in any medium, provided the original work is properly cited.

\begin{abstract}
The quality of palliative care given to terminally ill patients and their family members can be directly impacted by the attitudes that nurses hold towards palliative care. This study aimed to investigate the attitudes of nonpalliative care nurses towards death and dying in the context of palliative care. Nurses working within the medical aged care, cardiology and respiratory wards at two metropolitan teaching hospitals in Sydney completed the Frommelt Attitudes Towards Care of the Dying (FATCOD) scale, an anonymous self-administered questionnaire, and a twelve-item demographic questionnaire. A total of 95 completed surveys were used in the final analysis. The total FATCOD score was $119.8 \pm 11.1$, patient FATCOD was $79.6 \pm 8.6$, and family FATCOD was $40.2 \pm 4.4$. Of significance, the professional variables designation and role were associated with attitudes in the total FATCOD and country of birth, designation, highest level of education, and role were associated with attitudes towards the patient FATCOD. Scores for communication between the nurse and the terminally ill patient were poor. Health care facilities should focus on developing strategies to improve the communication skills among nonpalliative care nurses in order to optimize patient outcomes.
\end{abstract}

\section{Background}

Historically palliative care was delivered with the intention of minimizing the suffering faced by those with an incurable diagnosis of malignant disease [1]. However, palliative care has changed in more recent years, with it now being seen as an intervention for the delivery of quality end of life care to include those with a life-limiting, nonmalignant diagnosis [2]. A recent report published by the Australian Government showed that a total of 52,347 hospital separations in 20082009 were directly attributable to palliative care, a 56\% increase over the period of a decade [3]. While the majority of these palliative care separations were due to the primary diagnosis of cancer, other nonmalignant diseases, such as heart failure, chronic obstructive pulmonary disorder, renal failure, motor neuron disease, liver failure, and dementia/Alzheimer's disease, constituted $40 \%$ of all palliative care separations [3].
The overall purpose of palliative care is to reduce suffering by means of prevention, minimization, and relief, in order to improve the quality of life of both the patients who are experiencing a life-limiting illness and their family members [4]. A specific definition of palliative care is outlined by the Australian Institute of Health and Welfare in the National Health Data Dictionary and States.

Palliative care is care in which the clinical intent or treatment goal is primarily quality of life for a patient with an active, progressive disease with little or no prospect of cure. It is usually evidenced by an interdisciplinary assessment and/or management of the physical, psychological, emotional, and spiritual needs of the patient and a grief and bereavement support service for the patients and their carers/family [5].

The development and continued implementation of specialized palliative care teams throughout hospital systems 
both nationally and internationally has facilitated the delivery of such care in a high quality manner; however, it is acknowledged that the increasing number of people seeking palliative care will make it difficult for these teams to engage with every hospitalized, terminally ill patient and their family members [6]. As a result, engagement with the delivery of palliative care from all nursing staff within the acute care setting has become a necessity. To aid in the embedment of the palliative care philosophy in nursing practice, a stronger emphasis has been placed on the inclusion of palliative care training including caring for the dying in the curricula of both undergraduate and, where applicable, postgraduate university degrees as highlighted by the Australian Government's National Palliative Care Strategy and the development of the PCC4U (Palliative Care Curriculum for Undergraduates) program $[7,8]$. The inclusion of palliative care training in the undergraduate stage of a nurse's career is perceived to encourage the development of positive attitudes towards the provision of palliative care by reducing anxiety towards death and dying and preventing misconceptions about the care of terminally ill patients [9].

A review of the research literature suggests that the attitudes of nurses towards death and dying have a direct impact on the quality of palliative care delivered to terminally ill patients and their family members $[10,11]$. Several studies examining predictors of attitudes have indicated that the attitudes of nurses towards death and dying are highly influenced by factors including their age [12], education level $[13,14]$, years of nursing experience [14], job classification [14], education in end of life care $[10,12,14,15]$, and time spent caring for terminally ill patients $[15,16]$. The evidence however is not conclusive; for example, there is controversy in the literature concerning the impact of the age of nurses on attitudes towards death and dying. In a study, the recruited 355 inpatient and outpatient older oncology nurses demonstrated more positive attitudes compared to the younger nurses [17]. In another study however with 367 Japanese nurses working in convalescent homes, younger nurses demonstrated more positive attitudes compared to the older nurses [12]. In contrast, an Australian study that investigates 116 nurses working within a residential aged care organization found no association between age and attitudes towards death and dying [14]. Cultural differences in beliefs and values also play a role in the health care professionals' attitudes towards death and dying, thus impacting the provision of palliative care [18-20]. For example, in some cultures, family and community are deemed to be more important while the concepts of individuality and autonomy are important in western cultures $[18,20]$.

The majority of knowledge related to attitudes of nurses towards death and dying has been elicited from nursing staff working within settings where the palliative care philosophy is embedded into nursing practices [17]. There is a paucity of literature relating to the attitudes of nursing staff not working within palliative care, oncology, and residential care settings [17]. In addition, there is limited literature investigating whether cultural differences based on ethnicity impact nurses' attitudes towards palliative care.

\section{Aim}

The aim of this study was to investigate the attitudes of nonpalliative care nurses towards death and dying in the context of palliative care.

\section{Method}

This was a cross-sectional study that gathered data from August 2012 to September 2012, from nurses through an anonymous, self-administered questionnaire.

This study was conducted at two tertiary hospitals in Sydney, Australia. Nurses were eligible for inclusion if they worked in the aged care, cardiology and respiratory wards. Nursing staff of all designations including Nurse Unit Managers, Clinical Nurse Consultants, Clinical Nurse Educators, Registered Nurses, Enrolled Nurses, and Assistants in Nursing were invited to participate in the study. Staff members who were on annual leave, maternity leave, or long service leave at the commencement of the study period were excluded.

3.1. Data Collection. A letter of invitation explaining the aims and methods of the study and intention to publish results was sent out to prospective participants one week before the commencement of the study period. Information about the study was also provided during team meetings and in-services. Questionnaires were given to nursing staff for completion and the staff were required to place completed forms in a dedicated secure receptacle that was periodically collected by the investigators. Ethical approval was gained from the Local Health District Human Research Ethics Committee prior to commencement of the study.

Data were collected relating to demographics, professional characteristics, and attitudes towards death and dying. Demographic data collected included age, gender, country of birth, length of stay in Australia, ethnic background, and religious affiliations. Professional characteristics collected included type of ward, nursing designation, highest level of education attained, employment status, role, years of nursing experience, and previous palliative care training.

The Frommelt Attitudes Towards Nursing Care of the Dying (FATCOD) scale [10] was utilized to investigate the participants' attitudes towards death and dying. The FATCOD is a 30 -item scale with an equal number of positively and negatively worded items that are scored on a five-point Likert scale from "strongly disagree" to "strongly agree." Possible scores ranged from 30 to 150 with higher scores reflecting more positive attitudes. Twenty items in the FATCOD scale relate directly to the nurses' attitude towards the patient (possible range 20-100) and ten items relate directly to the nurses attitudes towards the patients' family (possible range 10-50).

An additional seven items that measured the nurses' attitudes towards death and dying were included in the questionnaire. Each of these items was positively worded and scored on a five-point Likert scale from "strongly disagree" to "strongly agree." The possible score for each of these items ranged from one to five, with higher scores reflecting 
more positive attitudes. The questions were sourced from two validated questionnaires $[21,22]$.

3.2. Data Analysis. All data was analyzed using IBM SPSS software (version 21). Negative items in the FATCOD scale were reverse-scored as per author guidelines [10]. Descriptive statistics were used to present demographic data and attitude scores. All demographic (age, gender, country of birth, length of stay in Australia, religious affiliation, and highest level of education) and professional characteristics (designation, employment status, role, and palliative care training) were included in a univariate analysis to determine each variable's individual relationship to nurse's attitudes overall (total FATCOD), towards the terminally ill patient (patient FATCOD), and towards the family of the terminally ill patient (family FATCOD). Statistical significance for all analyses was set at $P<0.05$.

\section{Results}

A total of 95 completed questionnaires were analyzed. The majority of respondents were below 50 years of age $(51.6 \%)$, were born outside of Australia (49.5\%), and were registered nurses (66.3\%). Fifty-six (58.9\%) respondents stated that their highest level of education was a Bachelor's degree and the majority of nurses worked on a full time basis (77.9\%) and in a clinical role $(87.4 \%)$. Less than $25 \%$ of the respondents had received palliative care training (Table 1 ).

4.1. Attitudes towards Death and Dying. The total FATCOD score achieved in this study was $119.8 \pm 11.1$ (range 30-150). In relation to attitudes of nurses towards the family members of terminally ill dying patients, the score was $40.2( \pm 4.4)$ out of a possible 50 and, in relation to attitudes towards the provision of care to patients, the score was $79.6( \pm 8.6)$ out of a possible 100. While the mean scores for most of the individual items in the FATCOD scale were equal to or greater than 4 (range 1-5), all the three items related specifically to communication between the nurse and terminally ill patients, that is, "I would be uncomfortable talking about impending death with the dying person," "the nurse should not be the one to talk about death with the dying person," and "when a patient asks 'Nurse am I dying?', I think it is best to change the subject to something cheerful," achieved lower mean scores, with scores ranging from 3.2 to 3.6. Mean scores for individual FATCOD items are presented in Figure 1. Cronbach's alpha reliability coefficient obtained in this study for the thirty-item FATCOD scale was 0.80 . Cronbach's alpha reliability coefficient for the patient related items was 0.81 and that for the family related items was 0.49 .

For the total FATCOD, significant relationships between nursing designation and attitudes towards death and dying were observed $(F=2.99 ; P=0.010)$. Similarly significant relationships between nursing role (clinical, educational, and managerial) and attitudes towards death and dying were identified $(F=7.15 ; P=0.001)$ (Table 2). For the patient related items within the FATCOD, country of birth, nursing designation, highest level of education, and role were also associated with attitudes towards death and dying
TABLE 1: Demographic characteristics of participants $(n=95)$.

\begin{tabular}{|c|c|}
\hline Variable & \\
\hline \multicolumn{2}{|l|}{ Age group (years), $n(\%)$} \\
\hline $20-29$ & $49(51.6)$ \\
\hline $30-39$ & $18(18.9)$ \\
\hline $40-49$ & $19(20.0)$ \\
\hline $50-59$ & $7(7.4)$ \\
\hline $60-69$ & $2(2.1)$ \\
\hline \multicolumn{2}{|l|}{ Gender, $n(\%)$} \\
\hline Male & $8(8.4)$ \\
\hline Female & $86(90.5)$ \\
\hline \multicolumn{2}{|l|}{ Country of birth, $n(\%)$} \\
\hline Australia & $43(45.3)$ \\
\hline Born outside Australia & $47(49.5)$ \\
\hline \multicolumn{2}{|l|}{ Nursing designation, $n(\%)$} \\
\hline Assistant in Nursing & $8(8.4)$ \\
\hline Endorsed Enrolled Nurse & $5(5.3)$ \\
\hline Registered Nurse & $63(66.3)$ \\
\hline Clinical Nurse Specialist & $6(6.3)$ \\
\hline Clinical Nurse Educator & $6(6.3)$ \\
\hline Clinical Nurse Consultant & $4(4.2)$ \\
\hline Nurse Unit Manager & $3(3.2)$ \\
\hline \multicolumn{2}{|l|}{ Highest level of education, $n(\%)$} \\
\hline Hospital certificate & $7(7.4)$ \\
\hline Associate diploma/diploma & $4(4.3)$ \\
\hline Bachelor's degree & $56(58.9)$ \\
\hline Graduate certificate/diploma & $15(15.8)$ \\
\hline Master's degree & $10(10.5)$ \\
\hline \multicolumn{2}{|l|}{ Employment status, $n(\%)$} \\
\hline Full time & $74(77.9)$ \\
\hline Part time & $20(21.1)$ \\
\hline \multicolumn{2}{|l|}{ Role, $n(\%)$} \\
\hline Clinical & $83(87.4)$ \\
\hline Education & $8(8.4)$ \\
\hline Managerial & $3(3.2)$ \\
\hline \multicolumn{2}{|l|}{ Palliative care training, $n(\%)$} \\
\hline No & $74(77.9)$ \\
\hline Yes & $19(20.0)$ \\
\hline \multicolumn{2}{|l|}{ Religious affiliation, $n(\%)$} \\
\hline No & $36(37.9)$ \\
\hline Yes & $58(61.1)$ \\
\hline Length of stay In Australia, years mean (SD) & $10.5(9.7)$ \\
\hline
\end{tabular}

(Table 2). No association was found between demographic or professional characteristics and family FATCOD attitude scores (Table 2).

Individual mean score calculations for the additional seven items included in the questionnaire to measure nurses attitudes towards palliative care are displayed in Table 3. Mean scores ranged from 2.6 to 3.2 . 


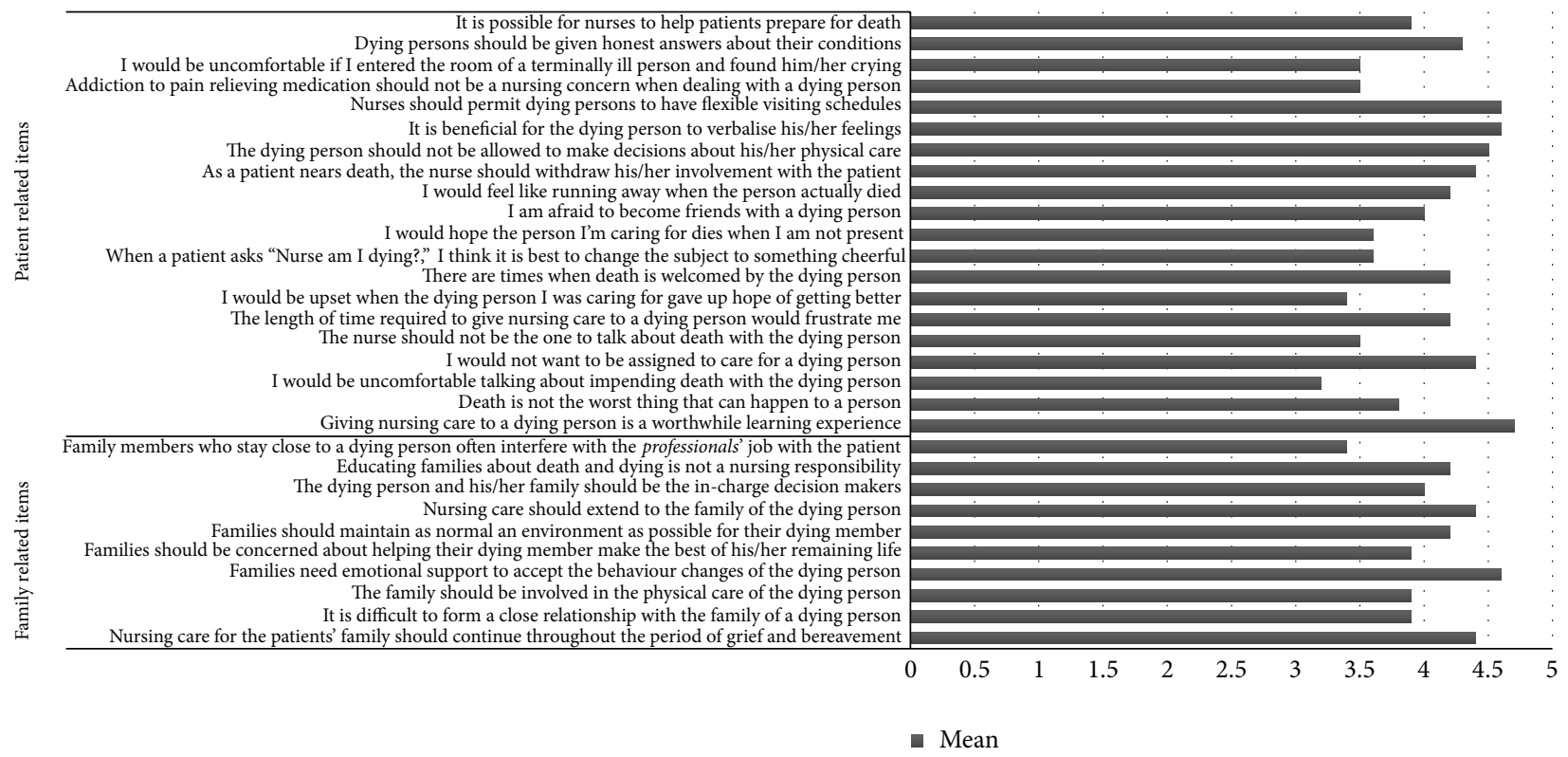

FIgURE 1: Mean scores for individual items of FATCOD scale.

TABLE 2: Associations between demographic/professional variables and attitudes towards death and dying.

\begin{tabular}{|c|c|c|c|c|}
\hline & \multicolumn{2}{|c|}{ Total FATCOD } & \multicolumn{2}{|c|}{ Patient FATCOD } \\
\hline & $F$ & $P$ & $F$ & $P$ \\
\hline Gender & 0.356 & 0.552 & 0.44 & 0.834 \\
\hline Age & 2.008 & 0.100 & 1.524 & 0.202 \\
\hline Country of birth & 1.205 & 0.269 & 16.738 & $0.000^{*}$ \\
\hline Years lived in Australia & 1.150 & 0.366 & 1.232 & 0.306 \\
\hline Ethnic background & 0.876 & 0.625 & 1.166 & 0.305 \\
\hline Nursing designation & 2.994 & $0.010^{*}$ & 4.420 & $0.001^{*}$ \\
\hline Highest level of education & 2.999 & 0.055 & 5.836 & $0.004^{*}$ \\
\hline Employment status & 3.415 & 0.068 & 3.513 & 0.64 \\
\hline Predominant role & 7.153 & $0.001^{*}$ & 8.805 & $0.000^{*}$ \\
\hline Years since first nursing qualification gained & 1.042 & 0.436 & 1.205 & 0.271 \\
\hline Palliative care training & 3.177 & 0.78 & 3.650 & 0.059 \\
\hline Religious affiliations & 0.582 & 0.477 & 1.504 & 0.223 \\
\hline
\end{tabular}

* Statistical significance.

TABLE 3: Mean scores (range 1-5) for seven additional attitudes items.

\begin{tabular}{ll}
\hline Attitude statement & Mean (SD) \\
\hline It is crucial for family members and significant others to remain at the bedside until death occurs & $3.2(1.1)$ \\
The provision of palliative care requires emotional detachment & $2.7(1.1)$ \\
Men generally reconcile their grief more quickly than women & $2.6(1.0)$ \\
The accumulation of losses renders burnout inevitable for those who seek work in palliative care & $2.9(0.8)$ \\
The loss of a distant or contentious relationship is easier to resolve than the loss of one that is close or intimate & $3.2(1.0)$ \\
Patients receiving palliative care must always be told they are dying & $3.0(1.0)$ \\
Religion and spirituality have the same meaning to patients in palliative care & $3.2(1.2)$ \\
\hline
\end{tabular}

\section{Discussion}

Increases in the demand for quality end of life care have seen palliative care highlighted as a national priority, as well as the progressively widespread provision of end of life care by nurses working in the acute medical and surgical hospital wards. In an environment where treatment with the aim of cure is the predominant end goal of care delivery, the growing expectation for nurses to embrace the palliative care philosophy of providing treatment with the aim of 
promoting quality of life in the grips of a life-limiting illness to facilitate a dignified and comfortable death provides a challenge. In order to align palliative care delivery with national goals "...to ensure that all Australians have access to quality palliative care and to ensure that no Australian with a terminal illness will suffer from preventable discomfort and pain" [23], it is important for palliative care leaders to assess hospital based nurses preparedness for integrating palliative care into their general practice. In the view that attitudes towards death and dying have a direct influence on the quality of the palliative care that is provided, the aim of this study was to explore the attitudes of nursing staff towards death and dying, in the context of palliative care provision, within an acute hospital setting.

The results of this study demonstrated that the nurses sampled have positive attitudes towards death and dying in the context of the delivery of palliative care to terminally ill patients and their families. In addition, nurses are uncomfortable with the notion of talking about death and dying with the terminally ill patient.

In this study, the attitudes of nurses towards death and dying were similar to those obtained in another Australian based study [14] that used the FATCOD scale and achieved a mean score of $119.8 \pm 11.1$. However, when compared to other published literature, the total FATCOD score in our study was higher than Turkish [13], Spanish [15], and Japanese [12] nurses, where the mean scores were $99.9 \pm 8.7$, $116.8 \pm 11.4$, and 107.2 \pm 12.1 , respectively. It is postulated that the overall higher scores obtained in this study may be due to the general acceptance of the palliative care philosophy within the two hospitals investigated. Specialised palliative care teams exist within both facilities and as part of their service these teams review all new palliative care referrals within 24 hours, allowing for the prompt patient assessment and documentation of end of life care plan and facilitating the provision of support for nursing and medical staff who may not be as experienced with caring for terminally ill, palliated patients. The ongoing, hospital based, palliative care education both formal and informal (real time teaching) provided by these teams may contribute also to the higher scores obtained in this study.

Interestingly, all the three items within the FATCOD scale that had low mean scores in comparison to the other individual items were related to communication with the terminally ill patient. These items were "I would be uncomfortable talking about impending death with the dying person," "the nurse should not be the one to talk about death with the dying person," and "when a patient asks 'Nurse am I dying?,' I think it is best to change the subject to something cheerful," suggesting that nursing staff are not entirely comfortable communicating with the terminally ill patients about their prognosis. It is therefore important that education carried out by the palliative care teams in the future should focus on the development of communication skills about death and dying between the nurse and the terminally ill patient. It is anticipated that development of these communication skills will improve the quality of palliative care that is delivered.

In this study, there was an association between demographic and professional characteristics and attitudes towards death and dying. Of significance, managerial and educational staff demonstrated higher scores compared to clinical staff in overall attitudes to palliative care (total FATCOD). It is difficult to determine why educational and managerial staff held more positive attitudes than clinical staff. It could be that nurses within the educational and managerial roles have had more exposure to the delivery of palliative care, a factor that was not investigated in this study. Nurses born within Australia had higher scores in patient related factors compared to nurses born outside Australia. Many cultural differences in relation to death and dying exist and such differences could be the reason why Australian born nurses had higher scores than those born outside Australia.

Limitations of this study include the small sample size and the exclusion of allied health and medical professionals who also form part of the multidisciplinary team that is necessary for the successful delivery of palliative care. A second limitation was the use of country of birth as a surrogate indicator of ethnicity which could bias the results. Despite these limitations, this study has provided valuable results and implications for nursing practice. Further research needs to be undertaken using large sample sizes that include multidisciplinary health care professionals. In addition, future research should also determine the participant's personal attitudes towards death, a variable that has been shown to influence attitudes towards caring for the terminally ill patient [11].

\section{Conclusion}

Nurses play an important role in the delivery of high quality palliative care within the hospital environment. Health care facilities should focus on developing strategies to improve the communication skills among nonpalliative care nurses in order to optimise patient outcomes.

\section{Conflict of Interests}

The authors declare that there is no conflict of interests regarding the publication of this paper.

\section{References}

[1] D. Clark, "From margins to centre: a review of the history of palliative care in cancer," The Lancet Oncology, vol. 8, no. 5, pp. 430-438, 2007.

[2] D. C. Traue and J. R. Ross, "Palliative care in non-malignant diseases," Journal of the Royal Society of Medicine, vol. 98, no. 11, pp. 503-506, 2005.

[3] Australian Institute of Health and Welfare, Trends in Palliative Care in Australian Hospitals, Australian Institute of Health and Welfare, 2011.

[4] World Health Organization, WHO Definition of Palliative Care, WHO, Geneva, Switzerland, 2014.

[5] Australian Institute of Health and Welfare, National Health Data Dictionary, Australian Institute of Health and Welfare, Canberra, Australia, 2012.

[6] B. A. Jack, M. Gambles, P. Saltmarsh, D. Murphy, T. Hutchison, and J. E. Ellershaw, "Enhancing hospital nurses knowledge 
of palliative care: a network nurse programme," International Journal of Palliative Nursing, vol. 10, no. 10, pp. 502-506, 2004.

[7] Department of Health, Supporting Australians to Live Well at the End of Life: National Pallaitve Care Strategy 2010, Department of Health, Canberra, Australia, 2010.

[8] Palliative Care Curriculum for Undergraduates Project Team, Principles for Including Pallaitve Care in Undergraduate Curricula, Queensland University of Technology, Brisbane, Australia, 2012.

[9] J. Cavaye and J. H. Watts, "An integrated literature review of death education in pre-registration nursing curricula: key themes," International Journal of Palliative Care, vol. 2014, Article ID 564619, 19 pages, 2014.

[10] K. H. M. Frommelt, “The effects of death education on nurses' attitudes toward caring for terminally ill persons and their families," The American Journal of Hospice and Palliative Medicine, vol. 8, no. 5, pp. 37-43, 1991.

[11] M. Braun, D. Gordon, and B. Uziely, "Associations between oncology nurses' attitudes toward death and caring for dying patients," Oncology Nursing Forum, vol. 37, no. 1, pp. E43-E49, 2010.

[12] M. Matsui and K. Braun, 'Nurses' and care workers' attitudes toward death and caring for dying older adults in Japan," International Journal of Palliative Nursing, vol. 16, no. 12, pp. 593-598, 2010.

[13] B. Cevik and K. Sultan, "Attitudes and experiences of nurses toward death and caring for dying patients in Turkey," Cancer Nursing, vol. 36, no. 6, pp. 1-8, 2013.

[14] R. Ford and F. McInerney, "An evaluation of aged-care workers' knowledge of and attitudes toward the palliative approach," Research in gerontological nursing, vol. 4, no. 4, pp. 251-259, 2011.

[15] T. M. Ho, E. Barbero, C. Hidalgo, and C. Camps, "Spanish nephrology nurses' views and attitudes towards caring for dying patients," Journal of Renal Care, vol. 36, no. 1, pp. 2-8, 2010.

[16] K. S. Dunn, C. Otten, and E. Stephens, "Nursing experience and the care of dying patients," Oncology Nursing Forum, vol. 32, no. 1, pp. 97-104, 2005.

[17] M. Lange, B. Thom, and N. E. Kline, "Assessing nurses' attitudes toward death and caring for dying patients in a comprehensive cancer center," Oncology Nursing Forum, vol. 35, no. 6, pp. 955959, 2008.

[18] K. Bullock, "The influence of culture on end-of-life decision making," Journal of Social Work in End-of-Life and Palliative Care, vol. 7, no. 1, pp. 83-98, 2011.

[19] G. B. Zaide, R. Pekmezaris, C. N. Nouryan et al., "Ethnicity, race, and advance directives in an inpatient palliative care consultation service," Palliative and Supportive Care, vol. 11, no. 1, pp. 5-11, 2013.

[20] K. Hiruy and L. Mwanri, "End-of-life experiences and expectations of Africans in Australia: cultural implications for palliative and hospice care," Nursing Ethics, vol. 21, no. 2, pp. 187-197, 2014.

[21] M. Proctor, L. Grealish, M. Coates, and P. Sears, "Nurses' knowledge of palliative care in the Australian Capital Territory," International Journal of Palliative Nursing, vol. 6, no. 9, pp. 421428, 2000.

[22] M. M. Ross, B. McDonald, and J. McGuinness, “The palliative care quiz for nursing (PCQN): the development of an instrument to measure nurses' knowledge of palliative care," Journal of Advanced Nursing, vol. 23, no. 1, pp. 126-137, 1996.

[23] Palliative Care Australia, National Palliative Care Consensus Statement, Palliative Care Australia, Canberra, Australia, 2011. 


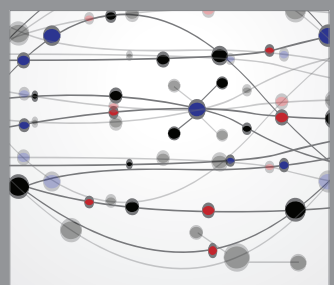

The Scientific World Journal
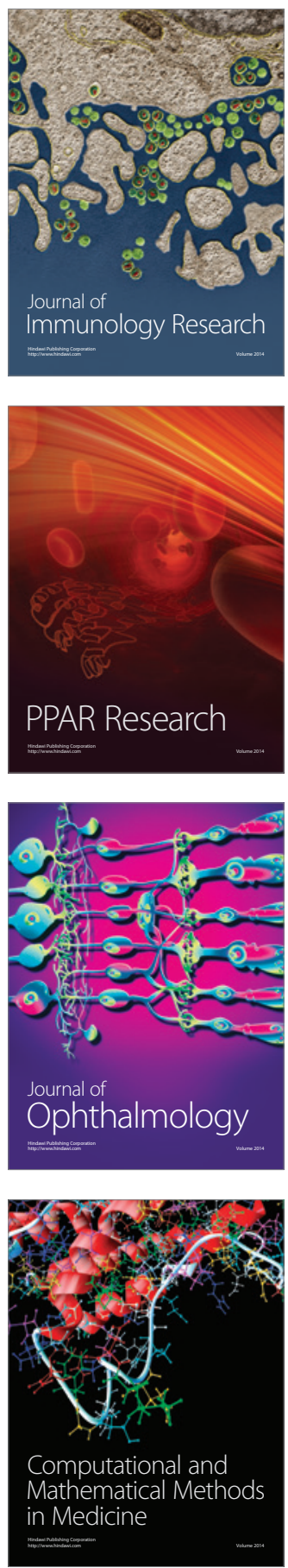

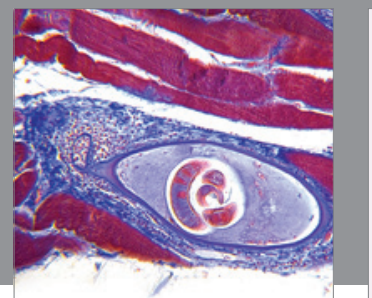

Gastroenterology

Research and Practice
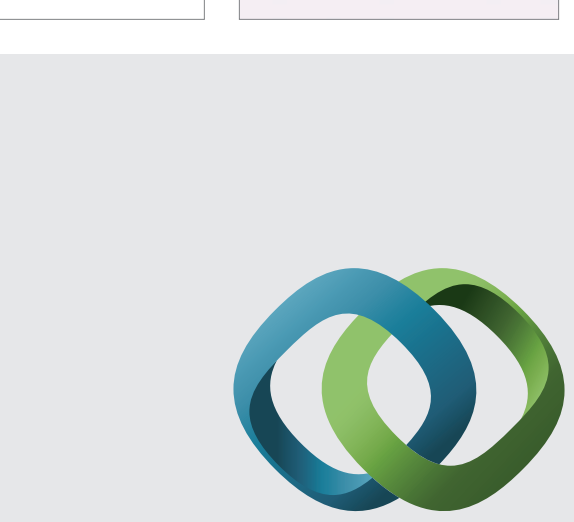

\section{Hindawi}

Submit your manuscripts at

http://www.hindawi.com
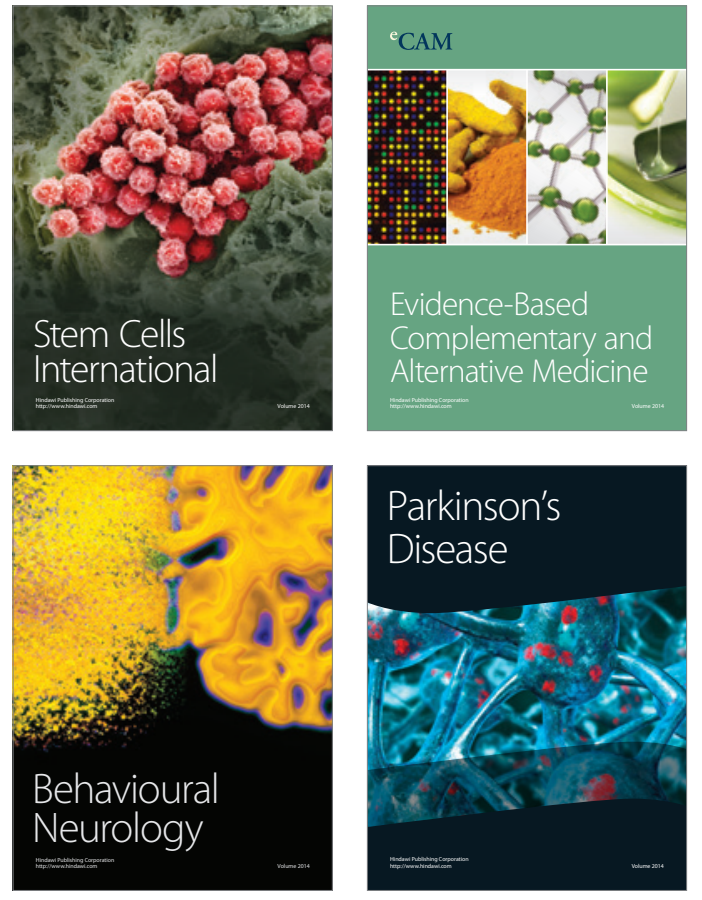
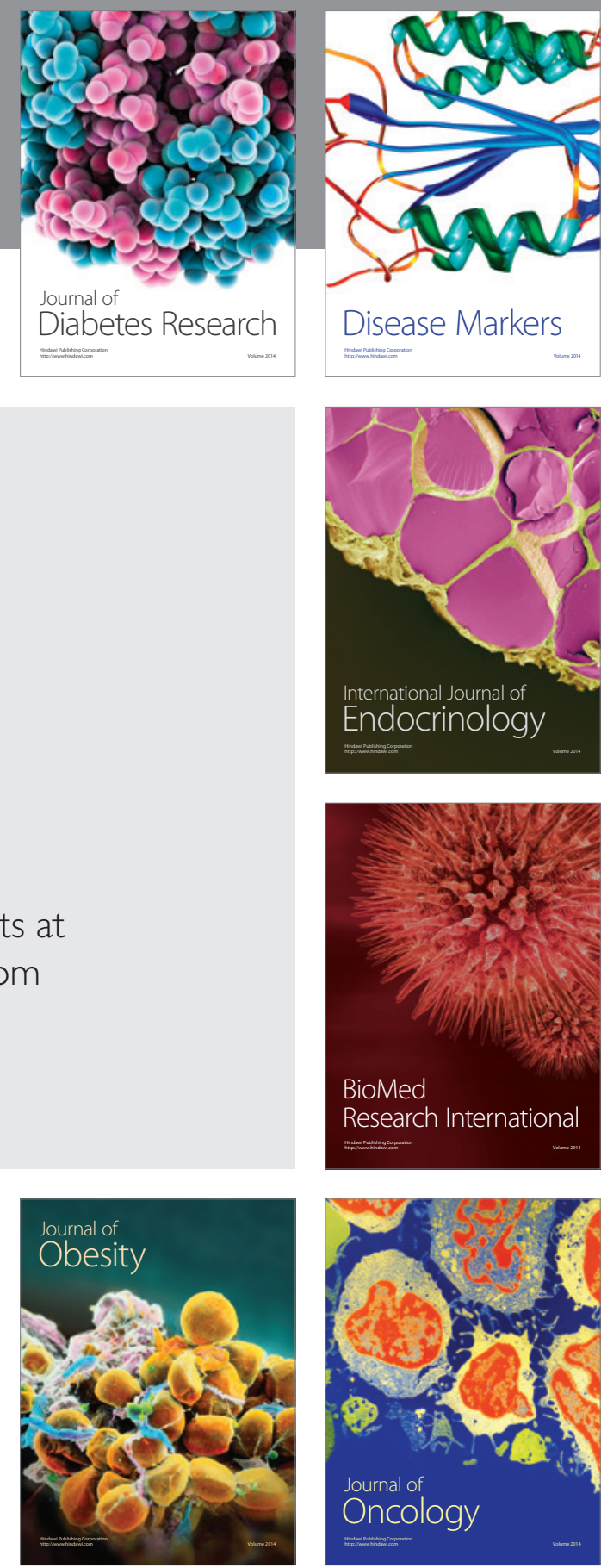

Disease Markers
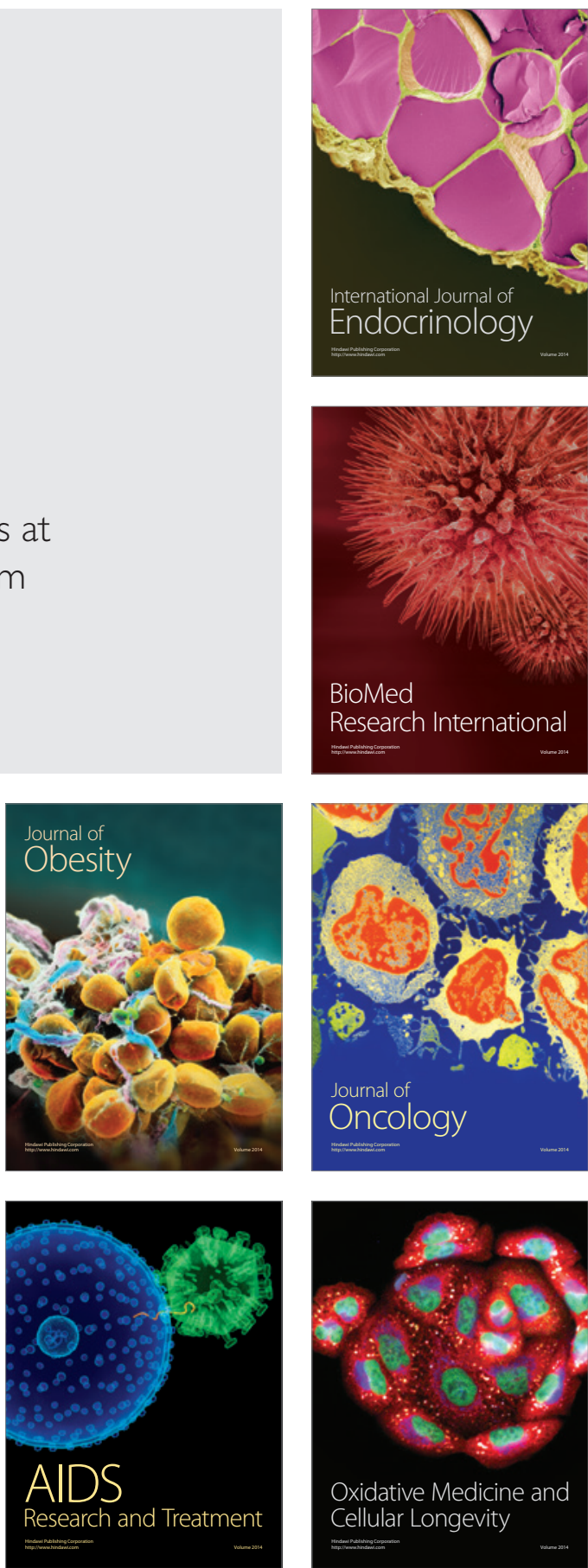